\title{
Enhancement of the scissors mode of an expanding Bose-Einstein condensate
}

\author{
M. Modugno, G. Modugno, G. Roati, C. Fort, and M. Inguscio \\ INFM - LENS - Dipartimento di Fisica, Università di Firenze \\ Via Nello Carrara 1, 50019 Sesto Fiorentino, Italy
}

(Dated: November 1, 2018)

\begin{abstract}
We study the time-evolution of the scissors mode of a Bose-Einstein condensate during the ballistic expansion after release from the magnetic trap. We show that despite the nontrivial character of the superfluid expansion, the sinusoidal behavior of the scissor oscillations is recovered after an asymptotic expansion, with an enhancement of the final amplitude. We investigate this phenomenon with a condensate held in an elongated magnetostatic potential, whose particular shape allows for the excitation of the scissors mode.

PACS numbers: 03.75.Fi, 05.30.Jp, 67.90.+z
\end{abstract}

The close connection between Bose-Einstein condensation in dilute atomic gases and superfluidity has been shown by recent research. The most striking signatures of superfluidity observed are quantized vortices [1], reduction of dissipative phenomena [2, 3], scissors modes [4, 5], and irrotational flow [6, 7].

Due to the particular requirements for the trapping potential, the observation of the scissors mode has been so far reported only for disk shaped condensates [5], where the superfluid behavior is evidenced by the different oscillation frequencies with respect to the thermal gas 伯. Very recently, the authors of [6, 7] have also analyzed the implication of irrotationality on the expansion of BoseEinstein condensates (BECs) initially confined in a rotating trap, showing that the condensate expands in a distinctively different way with respect to a non-rotating condensate, thus providing a direct manifestation of the quenching of the moment of inertia.

In this paper we show that the peculiar behavior of a free-expanding rotating condensate has also important consequences on the evolution of a scissors mode initially excited in the trap. Although the condensate undergoes a nontrivial expansion, we find that asymptotically the scissors mode recovers the initial sinusoidal behavior, with an enhancement of the final amplitude. To verify our predictions we take advantage of the particular shape of the magnetic field in a magnetostatic trap, which permits the excitations of scissors modes in elongated BECs. This allows us to investigate the expansion of a rotating superfluid in a novel regime, where the implications of the irrotationality are the most dramatic.

The wave function of a condensate at zero temperature can be conveniently written in terms of the density $\rho$ and the phase $S$

$$
\psi(\mathbf{x}, t)=\sqrt{\rho(\mathbf{x}, t)} e^{i S(\mathbf{x}, t) / \hbar} .
$$

In the Thomas-Fermi approximation the Gross-Pitaevskii equation for $\psi$ can be transformed in two coupled hydro-

\footnotetext{
*Also at: Dipartimento di Fisica, Università di Trento, 38050 Povo, Italy.
}

dynamics equations for the density $\rho$ and the velocity $\mathbf{v}=\nabla S / m[8,9$

$$
\begin{aligned}
& \frac{\partial \rho}{\partial t}+\nabla(\rho \mathbf{v})=0 \\
& m \frac{\partial \mathbf{v}}{\partial t}+\nabla\left(\frac{\mathbf{v}^{2}}{2 m}+U(\mathbf{r}, t)+g \rho\right)=0,
\end{aligned}
$$

where $U$ is the trapping potential, $g=4 \pi \hbar^{2} a / m$ the coupling strength, $m$ the atomic mass and $a$ the interatomic scattering length.

Here we are interested in the solution of Eqs. (2)-(3) which corresponds to a scissors mode, that is a shapepreserving oscillation of the condensate [4]. To account for this we consider a harmonic potential of the form

$$
U(\mathbf{x}, t) \equiv \frac{1}{2} m \omega_{h o}^{2} \sum_{i j=1}^{3} x_{i} W_{i j}(t) x_{j}
$$

where $W=W^{T}$ for symmetry reasons, with initial conditions $W_{i j}(t<0)=\omega_{i}^{2} \delta_{i j} / \omega_{h o}^{2}$, and $\omega_{h o} \equiv\left(\omega_{x} \omega_{y} \omega_{z}\right)^{1 / 3}$. By tilting the trap potential by a small angle $\theta_{0 i}$ around the $z$-axis at $t=0$, one can excite a scissors mode in the $x-y$ plane, identified by the angle

$$
\theta_{0}(t)=\theta_{0 i} \cos \left(\omega_{s c} t\right)
$$

whose frequency is $\omega_{s c}=\left(\omega_{x}^{2}+\omega_{y}^{2}\right)^{1 / 2}$ 田. In this case Eqs. (2)-(3) can be solved exactly with a quadratic ansatz for the condensate density and phase [9, 10

$$
\begin{aligned}
& \rho(\mathbf{x}, t)=\frac{m \omega_{h o}^{2}}{g}\left(\rho_{0}(t)-\frac{1}{2} \sum_{i, j=1}^{3} x_{i} A_{i j}(t) x_{j}\right), \\
& S(\mathbf{x}, t)=m \omega_{h o}\left(s_{0}(t)+\frac{1}{2} \sum_{i, j=1}^{3} x_{i} B_{i j}(t) x_{j}\right) .
\end{aligned}
$$

Therefore, in general the system can be described by 10 time dependent dimensionless parameters: $\rho_{0}, S_{0}, A_{i j}$ and $B_{i j}$ ( $A$ and $B$ are $3 \times 3$ symmetric matrices, reflecting the symmetry property of $U$, with $A_{i 3}=0=B_{i 3}$ due 
to the vanishing of $\left.W_{i 3}(t)\right)$. These parameters obey the following set of first order differential equations 10 .

$$
\begin{aligned}
& \frac{d \rho_{0}}{d \tau}=\rho_{0} \operatorname{Tr} B ; \frac{d S_{0}}{d \tau}=\rho_{0} \\
& \frac{d A}{d \tau}=2 A \operatorname{Tr} B+\{A, B\} \\
& \frac{d B}{d \tau}=W-A-B^{2}
\end{aligned}
$$

with boundary conditions determined by the equilibrium distribution of the condensate at $\tau \equiv \omega_{h o} t=0$. Notice that these equations depend on the number of atoms $N$ and on the scattering length $a$ only through the initial value of $\rho_{0}(0)=0.5 a_{h o}^{2}\left(15 \mathrm{Na} / \mathrm{a}_{h o}\right)^{2 / 5}$.

The subsequent angular motion is evaluated in terms of the rotation which diagonalizes the quadratic part of the density $\rho(\mathbf{x}, t)$, (that is the matrix $A_{i j}$ ). The angle of rotation is fixed by the relation [6]

$$
\tan \left(2 \theta_{0}\right)=\frac{2 A_{12}}{A_{11}-A_{22}} .
$$

The same Eqs. (8)-(10) can be used to study the expansion of the condensate after the release from the trap $(W \equiv 0)$, with the appropriate initial conditions.

Experimentally we study the scissors mode of a BEC of ${ }^{87} \mathrm{Rb}$ atoms in an elongated potential created by a QUIC magnetostatic trap 111. The potential has a cylindrical symmetry around the $x$-axis, with frequencies $\omega_{x}=2 \pi \times 16.3 \mathrm{~Hz}$ and $\omega_{y}=\omega_{z}=2 \pi \times 200 \mathrm{~Hz}$. The BEC is produced by standard radio-frequency evaporation, and is typically composed by $10^{5}$ atoms. In all the measurements we report no thermal component was detectable.

Since the trap is static, the scissors mode cannot be excited with the technique demonstrated for time-orbitingpotential traps [5]. However, we can take advantage of the fact that gravity breaks the symmetry of the magnetic potential resulting in anharmonicity even at the trap minimum. Actually, we found that the eigenaxes of our magnetic trap are rotated in the $x-y$ plane in a localized region around the minimum. The rapid motion of the condensate through such deformation is therefore equivalent to a sudden rotation of the trap, like that considered in Eq. (5). In fact, we found that when we excite a dipolar oscillation along the weak $x$-axis by a sudden displacement of the trap minimum, a scissors mode appears as soon as the BEC travels for the first time through the minimum. Since for an elongated BEC the scissors mode proceeds essentially at $\omega_{y} \gg \omega_{x}$, it is possible to study several scissors oscillations before the BEC travels again through the center of the trap. By choosing an appropriately small dipolar amplitude, we observe a "pure" scissors mode with amplitude $\theta_{0 i} \simeq 15 \mathrm{mrad}$, without any apparent shape deformation.

To describe the characterization of such mode, we need first to recall the peculiar behavior of a rotating BEC during the expansion after release from the trap [6]. In fact, due to the irrotational nature of a superfluid $(\nabla \times \mathbf{v}=0)$

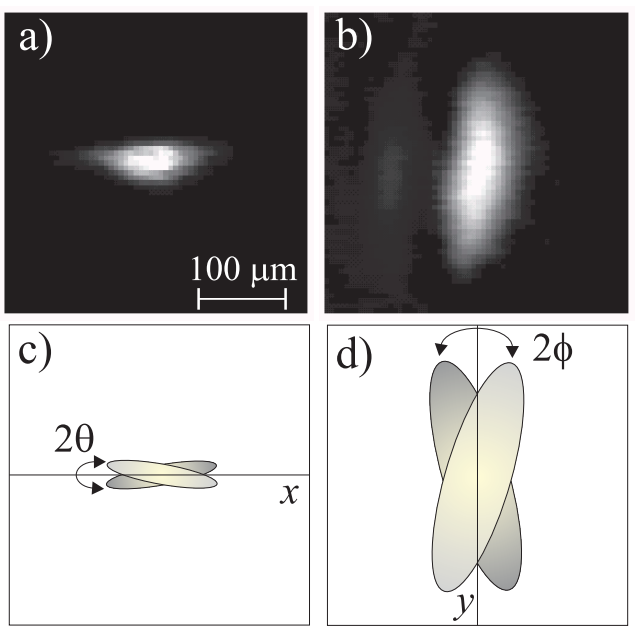

FIG. 1: Absorption images of a rotating, elongated Rb condensate during the ballistic expansion. In a) the BEC has just been released from the trap $\left(t_{\exp }=2 \mathrm{~ms}\right)$, and in b) it has expanded for $23 \mathrm{~ms}$. The other two pictures, not to scale, show the corresponding evolution of a scissors mode. The initial oscillation about the horizontal $x$-axis (c) is transformed in an oscillation about the vertical $y$-axis for long expansion times (d).

the moment of inertia $\Theta$ of a condensate is quenched with respect to the rigid body value $\Theta_{\text {rig }}$ 12.

$$
\Theta=\frac{\left\langle x^{2}-y^{2}\right\rangle^{2}}{\left\langle x^{2}+y^{2}\right\rangle^{2}} \Theta_{r i g}
$$

Therefore, due to conservation of energy and angular momentum, a rotating condensate cannot reach a symmetric configuration during the expansion, and undergoes a rapid rotation causing the inversion of the "long" and "short" axes. In particular, if at release the condensate is rotating in the $x-y$ plane, with its long axis forming a small angle $\theta_{0}$ with the $x$-axis, as shown in Fig. 1, initially it will start expanding in the short direction. Then, when the aspect ratio approaches unity, the condensate will continue to expand in the long direction, with its long axis rotated by an angle which is close to but not exactly $\pi / 2$ [4].

We have studied the evolution of the rotation angle $\theta$ of the condensate as a function of the expansion time $t_{\text {exp }}$, as shown in Fig. 2(a). The measurements are performed by releasing the condensate after a fixed evolution time $t$ of the scissors oscillations in the trap. For comparison we also plot the angle evolution of the thermal cloud just above condensation, which has been put under rotation with the same technique described above. Note that the rotation of the thermal cloud can be experimentally investigated only until the cloud becomes spherical at $t_{\exp } \simeq 1 / \omega_{x}$. Initially, the expansion of the BEC and the thermal cloud is almost indistinguishable, being characterized by the angular velocity $\Omega_{0}$ at release from the trap. This is due to the fact that the moment of inertia of an elongated condensate is close to the rigid body 


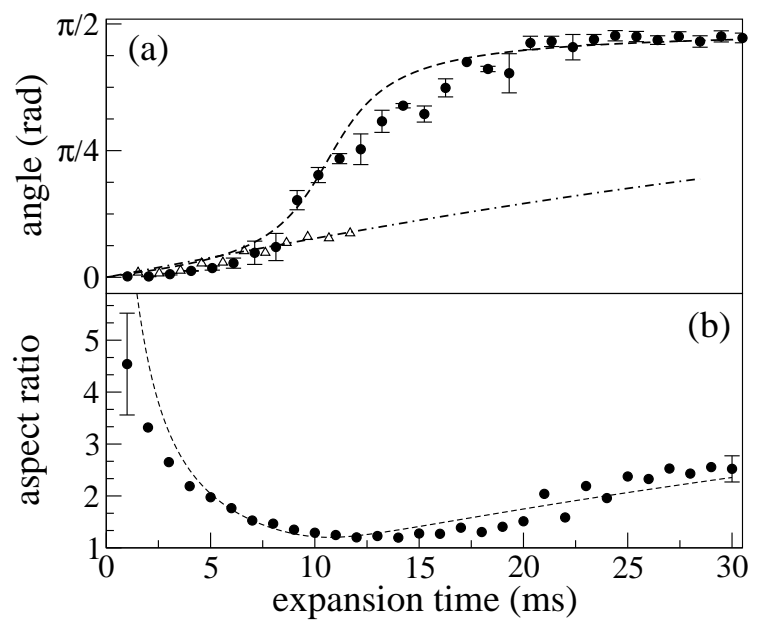

FIG. 2: a) Evolution of the rotation angle for a condensate (circles) and a thermal cloud (triangles) released with small angular velocity from an elongated trap. b) Evolution of the aspect ratio of the rotating condensate. When the aspect ratio gets close to unity the angular velocity shows a rapid increase. The lines represent the theoretical predictions.

value (see Eq. (12)). The quenching becomes important as soon as the aspect ratio approaches unity, where the non-classical behavior of the condensate is evident, as predicted in [6]. Indeed, its rotation undergoes a fast acceleration (Fig. 2(b)), followed by a slow evolution towards an asymptotic angle, close but smaller than $\pi / 2$. The agreement of experiment and theory is quite good. In contrast, the rotation angle of the thermal cloud continues to follow the predicted behavior [6]

$$
\theta\left(t ; t_{\text {exp }}\right)=\theta_{0}(t)+\operatorname{atan}\left(\Omega_{0}(t) t_{\text {exp }}\right),
$$

as shown in Fig. 2(a). This figure shows that the use of very elongated condensates allows for the investigation of novel regimes where the superfluid expansion is dramatically different from the classical one.

By analyzing the expansion of the condensate for the various conditions of initial angle and angular velocity at release from the trap, it is possible to reconstruct the evolution of a scissors oscillation for the expanding BEC. As an example, in Fig. 3 we show the calculated evolution of the angle $\theta$ during the time of flight, for conditions corresponding to very small angular velocity and maximal angular velocity of the trapped oscillations. The behavior of $\theta$ in all these regimes is qualitatively similar to that shown in Fig. 2. However, in the situations of small initial angular velocity the transient rotation is even faster than in the other cases, while the asymptotic angle is closer to $\pi / 2$.

By studying the solutions of Eq. (8)-(10), we can identify three different regimes for the expanding scissors mode, depending on the expansion time $t_{\text {exp }}$. i) For $t_{\text {exp }}<1 / \omega_{x}$ the condensate expands in a way similar to a classical gas, and the sinusoidal behavior of the scissors mode is preserved, as is possible to see from Eq. (13).

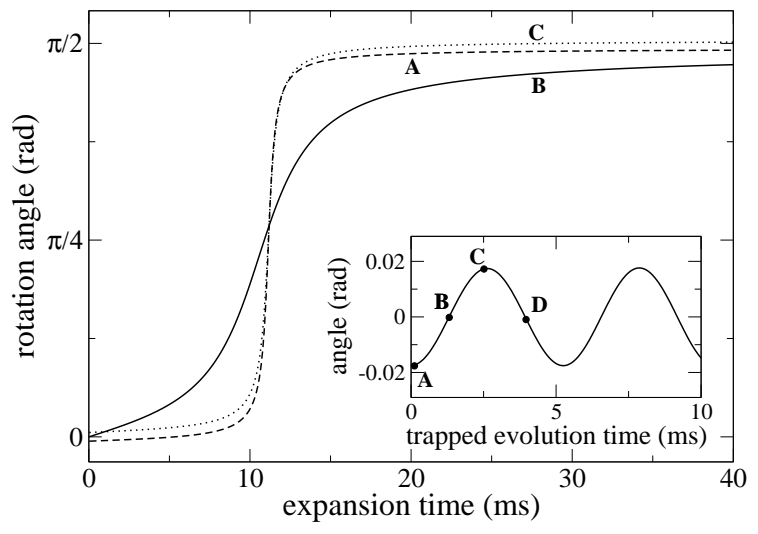

FIG. 3: Calculated rotation angle of the condensate during the expansion, released at different times during the scissors oscillation in the trap, as shown in the inset. Cases A and C correspond to small angular velocities, B and D to the maximum velocity. The evolution of $\mathrm{D}$ is the opposite of $\mathrm{B}$ (negative angles).

ii) When $t_{\text {exp }} \simeq 1 / \omega_{x}$ the rotation angle is always close to $\pm \pi / 4$, depending on the sign of the angular velocity at release. The angle oscillation is therefore close to a square wave with the same phase of the angular velocity in the trap $\Omega_{0}(t)$. iii) For $t_{e x p}>1 / \omega_{x}$ the angle of the condensate approaches $\pi / 2$, and it is more convenient to study the oscillations of the angle $\phi$ from vertical. We find that the original sinusoidal behavior of the oscillations is restored, with a time-dependence which can be fitted by the form

$$
\begin{aligned}
\phi\left(t ; t_{\text {exp }}\right) & =\theta_{0}(t)+F\left(t_{\text {exp }}\right) \Omega_{0}(t) \\
& =\theta_{0 i} \sqrt{1+\omega_{s c}^{2} F^{2}\left(t_{e x p}\right)} \cos \left(\omega_{s c} t+\varphi\right),
\end{aligned}
$$

where $F$ is a non-trivial function of the expansion time and of the trap geometry, and $\varphi$ is a phase shift. This behavior is remarkable, since in passing over the "critical" time-region at $t_{\text {exp }} \simeq 1 / \omega_{x}$, the condensate does not lose memory of its initial angular velocity, and it starts to behave again as a classical gas.

We have been able to verify experimentally these expectations in our Rb BEC. Since the finite resolution of the imaging system prevents us from studying the condensate inside the trap, we probe the oscillation of the condensate after a minimum expansion $t_{\text {exp }}=4 \mathrm{~ms}$, as shown in Fig. 4(a), and we reconstruct the scissors mode in the trap. In Fig. 1(b) we compare such a motion to the experimental scissors mode after a long expansion of $23 \mathrm{~ms}$. The initial scissors mode is clearly amplified and phase-shifted after the expansion. For the experimental parameters we calculate indeed that $\omega_{s c} F\left(t_{e x p}=23 \mathrm{~ms}\right) \simeq 10.7$ is much larger than one, and therefore the time evolution of the scissors mode is substantially a replica of that of the angular velocity in the trap. We have observed a similar behavior also for collision-induced scissors oscillations of a binary BEC system 13. 


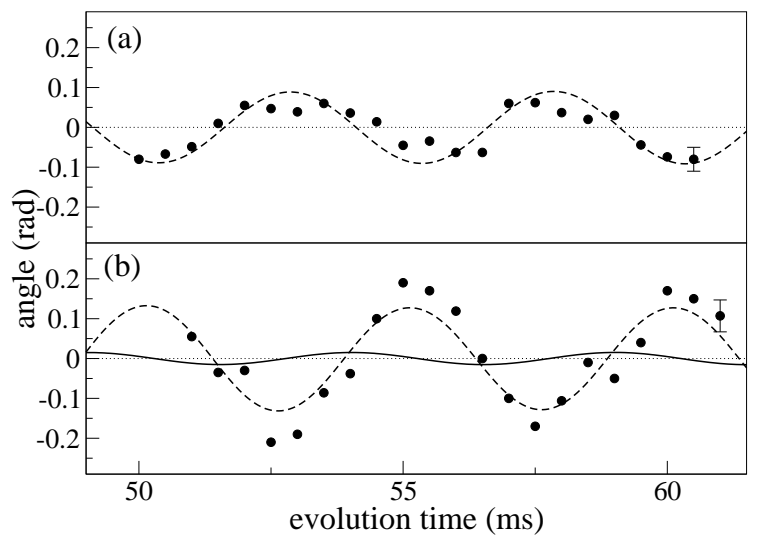

FIG. 4: Evolution of the scissors mode during the ballistic expansion. In a) the angle oscillation $\theta(t)$ after a short expansion of $4 \mathrm{~ms}$ (circles) is compared to theory (dashed line) to extrapolate the oscillation in the trap. In b) we compare the latter (continuous line) to the oscillation $\phi(t)$ after a longer expansion of $23 \mathrm{~ms}$ (circles). The data show an enhancement of the scissors mode, as expected from theory (dashed line).

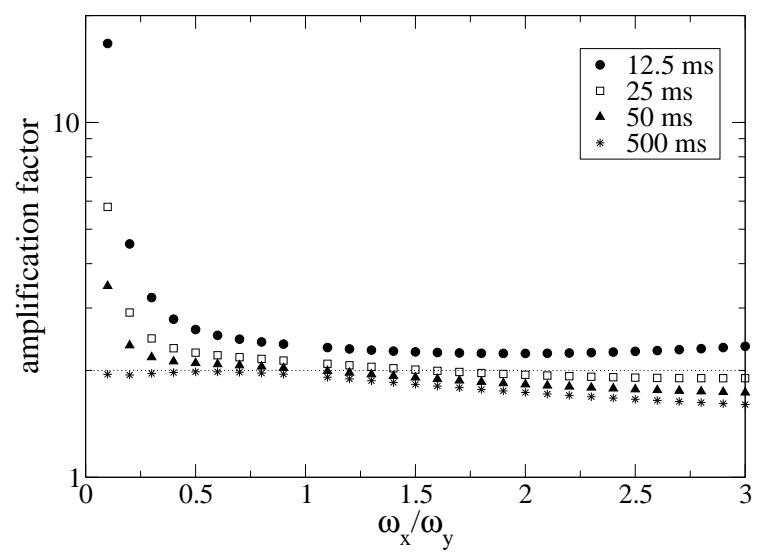

FIG. 5: Calculated amplification factor $\sqrt{1+\omega_{s c}^{2} F^{2}}$ of a scissors mode as a function of $\omega_{x} / \omega_{y}$, for various expansion times: $t_{e x p}=25,50,100,500 \mathrm{~ms}$. Here the the scissor frequency is kept fixed to $\omega_{s c}=2 \pi \times 200.7 \mathrm{~Hz}$ and the radial and axial frequencies are varied accordingly.

[1] M. R. Matthews, et al., Phys. Rev. Lett. 83, 2498 (1999); K. W. Madison, et al., Phys. Rev. Lett. 84, 806 (2000).

[2] C. Raman, et al., Phys. Rev. Lett. 83, 2502 (1999).

[3] S. Burger, et al., Phys. Rev. Lett. 86, 4447 (2001).

[4] D. Guery-Odelin and S. Stringari, Phys. Rev. Lett. 83, 4452 (1999).

[5] O. M. Maragò, S. A. Hopkins, J. Artl, E. Hodby, G. Heshenblaikner, and C. J. Foot Phys. Rev. Lett. 84, 2056 (2000); O. M. Maragò, G. Heshenblaikner, E. Hodby, S. A. Hopkins, and C. J. Foot, cond-mat/0109193.

[6] M. Edwards, C. W. Clark, P. Pedri, L. Pitaevskii, and S. Stringari, Phys. Rev. Lett. 88, 070405 (2002).
To study the role played by the trap geometry in the amplification mechanism, we have calculated the ratio between the final amplitude and the trapped one as a function of the trap anisotropy $\omega_{x} / \omega_{y}$, for various expansion times. In Fig. 5 we show the results, obtained by keeping fixed the scissor frequency to the value $2 \pi \times 200.7 \mathrm{~Hz}$, and varying the radial and axial frequencies accordingly. The behavior shown is independent of the initial angle $\theta_{0 i}$, in the small angle regime. This picture confirms that in cigar-shaped traps, for typical expansion times $\left(t_{\text {exp }} \simeq 10 \div 30 \mathrm{~ms}\right.$ ), the scissors mode amplitude is substantially amplified by the expansion. It also shows that the amplification factor asymptotically tends to a value $\sim 2$, regardless the trap geometry. It is worth noticing that this asymptotic behavior is similar to that of a $2 \mathrm{D}$ condensate in the limit $\omega_{x} / \omega_{y}=1$, where the relation (14) can be demonstrated analytically [14]. Notice that in contrast Eq. (13) would not imply any amplification of the asymptotic oscillations of the thermal cloud.

As a final remark we recall that in general the scissors modes are coupled to compressional modes, since a rotation of the condensate can induce shape deformation if the rotation angle is not small enough [4]. In our trap, by increasing the amplitude of the dipolar oscillation, and hence the angular velocity acquired by the condensate, it is also possible to access the non-linear regime, where the scissors mode couples to quadrupole shape oscillations.

In conclusion, we have investigated the expansion of the scissors mode of BECs. We have shown that the asymptotic oscillation is still characterized by a sinusoidal behavior, which depends linearly on the initial amplitude and angular velocity, resulting in an enhancement of the final amplitude. The capability of exciting scissors oscillations in elongated condensates opens the possibility of future studies of rotating superfluids in a novel regime of large moments of inertia and small angular velocities.

We acknowledge useful discussion with F. S. Cataliotti, P. Pedri, and S. Stringari, and the helpful contribution by L. Fallani. This work was supported by MIUR, by ECC under the Contract HPRICT1999-00111, and by INFM, PRA "Photonmatter".
[7] G. Heshenblaikner, E. Hodby, S. A. Hopkins, O. M. Maragò, and C. J. Foot, Phys. Rev. Lett. 88, 070406 (2002).

[8] S. Stringari, Phys. Rev. Lett. 77, 2360 (1996).

[9] F. Dalfovo, et al., Rev. Mod. Phys. 71, 463 (1999).

[10] F. Dalfovo, C. Minniti, and L. P. Pitaevskii, Phys. Rev. A 56, 4855 (1997); S. Sinha and Y. Castin, Phys. Rev. Lett. 87, 190402 (2001).

[11] For more details on the experimental apparatus see: G. Modugno, G. Ferrari, G. Roati, R. J. Brecha, A. Simoni, and M. Inguscio, Science 294, 1320 (2001).

[12] F. Zambelli and S. Stringari, Phys. Rev. A 63, 033602 
(2001).

[14] Y. Castin and R. Dum, Eur. Phys. J. D 7, 399 (1999).

[13] G. Modugno, M. Modugno, F. Riboli, G. Roati, and M. Inguscio, preprint cond-mat/0205485. 